\title{
Overexpression of IFITM3 Predicts the High Risk of Lymphatic Metastatic Recurrence in pNO Esophageal Squamous Cell Carcinoma after Ivor-Lewis Esophagectomy
}

Yang Jia, Miao Zhang, Wenpeng Jiang, Zhiping Zhang, Shiting Huang, Zhou Wang

Background: Recent studies have shown that the aberrant expression of IFITM3 is implicated in the lymph node metastasis of many malignancies. Our research aimed to investigate the expression of IFITM3 in pathological NO (pNO) esophageal squamous cell carcinoma (ESCC) and its relationship with lymph node metastatic recurrence. Methods: Immunohistochemistry (IHC) was used to examine the expression profile of IFITM3 in 104 pairs of samples. Each pair consisted of ESCC tissue and its adjacent normal mucosa (ANM). This aberrant expression was verified by reverse transcription-polymerase chain reaction (RT-PCR) with 20 tumor specimens with strong immunostaining and their mucosal tissues. Inaddition, 20 samples of low expression tissues and their ANMs were evaluated. Moreover, the correlations between the IFITM3 expression level and the clinicopathological variables, recurrent status and overall survival (OS) of patients were analyzed. Results: Both IHC and RT-PCR demonstrated that the IFITM3 expression level was significantly higher in tumor tissue than in ANM. Statistical analysis showed a significant correlation of IFITM3 expression with the T status of esophageal cancer $(p=0.015)$. Inaddition, IFITM3 overexpression was demonstrated to be not only an important risk factor of lymphatic metastatic recurrence but a significant prognostic factor in pNO ESCC $(p<0.005)$.

Conclusions: Even pNO ESCC patients will still experience lymphatic metastatic recurrence. The IFITM3 gene could be a predictor of lymphatic metastatic recurrence in pNO ESCC after Ivor-Lewis esophagectomy. 
1 Overexpression of IFITM3 Predicts the High Risk of Lymphatic

2 Metastatic Recurrence in pNO Esophageal Squamous Cell

3 Carcinoma after Ivor-Lewis Esophagectomy

\section{Abstract}

5 Background: Recent studies have shown that the aberrant expression of IFITM3 is implicated in

6 the lymph node metastasis of many malignancies. Our research aimed to investigate the

7 expression of IFITM3 in pathological N0 (pN0) esophageal squamous cell carcinoma (ESCC)

8 and its relationship with lymph node metastatic recurrence.

9 Methods: Immunohistochemistry (IHC) was used to examine the expression profile of IFITM3

10 in 104 pairs of samples. Each pair consisted of ESCC tissue and its adjacent normal mucosa

11 (ANM). This aberrant expression was verified by reverse transcription-polymerase chain

12 reaction (RT-PCR) with 20 tumor specimens with strong immunostaining and their mucosal

13 tissues. In addition, 20 samples of low expression tissues and their ANMs were evaluated.

14 Moreover, the correlations between the IFITM3 expression level and the clinicopathological

15 variables, recurrent status and overall survival (OS) of patients were analyzed.

Results: Both IHC and RT-PCR demonstrated that the IFITM3 expression level was

17 significantly higher in tumor tissue than in ANM. Statistical analysis showed a significant correlation of IFITM3 expression with the T status of esophageal cancer $(p=0.015)$. In addition, 
19 IFITM3 overexpression was demonstrated to be not only an important risk factor of lymphatic

20 metastatic recurrence but a significant prognostic factor in pN0 $\operatorname{ESCC}(p<0.005)$.

21 Conclusions: Even pN0 ESCC patients will still experience lymphatic metastatic recurrence.

22 The IFITM3 gene could be a predictor of lymphatic metastatic recurrence in pN0 ESCC after

23 Ivor-Lewis esophagectomy.

24 Authors: Yang $\mathrm{Jia}^{1}$, Miao Zhang ${ }^{2}$, Wenpeng Jiang ${ }^{1}$, Zhiping Zhang ${ }^{1}$, Shiting Huang ${ }^{2}$, Zhou

25 Wang $^{1 *}$

261 Department of Thoracic Surgery, Provincial Hospital Affiliated to Shandong University, Jinan,

27 Shandong, China;

282 Shandong Medical Imaging Research Institute, Shandong University, Jinan, China;

29 * Professor Wang Zhou, to whom correspondence should be addressed. Department of Thoracic

30 Surgery, Provincial Hospital Affiliated to Shandong University, Jinan, Shandong, China

31 Tel: 0086-0531-87938911(B); 0086-13665312567(M)

32 Email: wz620226@hotmail.com 


\section{Introduction} geographic distribution, and China is a country with high incidence of ESCC (Ferlay et al. 2010).

Despite the improvement in the diagnostic level and utilization of combined treatment modalities in recent years, the prognosis of ESCC patients remains poor. Even in pN0 ESCC, the 5-year survival rate is only approximately $70 \%$ after complete resection, and lymphatic metastatic recurrence is the main reason for the failure of the operation (Eloubeidi et al. 2002; Visbal et al.

51 2001). Therefore, to improve the long-term survival of ESCC patients, it is of great clinical

52 significance to control locoregional lymph node metastatic recurrence after surgery. 
53 Clinically, the number of metastasis-positive lymph nodes is usually used to evaluate the

54 risk of lymphatic metastatic recurrence in locally advanced disease (Law \& Wong 2001).

55 However, no reliable index has been used to predict this rate in pN0 ESCC. Although some

56 molecules were previously used to stratify the recurrent risk in pN0 ESCC (Li et al. 2009; Song

57 et al. 2012), none has been proven to be universally accepted and commonly used. Therefore,

58 sensitive biological markers that may predict this recurrent risk are urgently needed.

Interferon-induced transmembrane protein 3 (IFITM3), also known as 1-8U, is one of the important members of the IFN-inducible transmembrane protein family. IFITM3 likely exerts

61 profound influences on cell proliferation, migration, and invasion through the modulation of the

62 Wnt/ $\beta$-catenin signaling pathway and is implicated in the G0/G1 checkpoint to control the cell

63 cycle of tumors (Hu et al. 2014; Yang et al. 2013; Zhao et al. 2013). It is overexpressed in many

64 human malignancies, such as gastric cancer, colorectal tumor, breast cancer, glioma, and oral

65 squamous cell carcinoma.

66 Previous research has shown that IFITM3 is upregulated in gastric cancer, which is

67 correlated with tumor invasion and metastasis (Hu et al. 2014). Moreover, it has also been demonstrated to have a close relationship with the prognosis of colon cancer and was confirmed to be an independent risk factor for disease-free interval ( $\mathrm{Li}$ et al. 2011). However, the clinicopathological significance and prognostic value of IFITM3 in ESCC patients remains

71 unknown. 
In this study, we sought to validate the relationships between the expression level of

IFITM3 and the clinicopathological characteristics, lymphatic metastatic recurrence risk and

overall survival of ESCC patients who had undergone Ivor-Lewis esophagectomy. We aimed to

explore whether the IFITM3 gene can predict lymphatic metastatic recurrence in pN0 ESCC.

\section{Materials and Methods}

\section{Patients}

From January 2008 to January 2010, patients with midthoracic ESCC who underwent IvorLewis esophagectomy with two-field lymphadenectomy in our department (Provincial Hospital Affiliated to Shandong University, China) were eligible for this study. In total, this study enrolled 104 patients, including 83 men and 21 women, with ages ranging from 40 to 75 years (Clinicopathological data are listed in Table 1).

All patients met the following inclusion criteria: (1) According to 2009 Union for International Cancer Control (UICC) standard for midthoracic ESCC, Ivor-Lewis esophagectomy with two-field lymph node dissection was conducted to achieve complete resection (R0), and the proximal and distal incisal margins as well as lateral margin were pathologically examined without residual foci (Arai et al. 2012). At the same time, the average number of lymph nodes dissected was $18 \pm 5.8$ (ranging from 12 to 25); (2) Patients enrolled in the study were restaged after surgery according to TNM staging (UICC, 2009) for esophageal cancer; (3) Without history of previous malignancies or other severe diseases that may influence 
91 the outcome of our follow-up; (4) Patients were not eligible if preoperative neoadjuvant

92 chemotherapy or postoperative adjuvant treatment was administered.

\section{Surgical procedure of Ivor-Lewis esophagectomy}

94 Four thoracic surgeons worked together to perform this type of surgery, and the thoracic operation was performed by two surgeons. The patient was placed in the $40^{\circ}-45^{\circ}$ left lateral decubitus position. After a right anterolateral thoracotomy, the chest was entered through the fourth intercostal space. The azygos vein arch was divided, and the esophagus was dissected from the esophagogastric junction to the apex of the chest. When the tumor invasion obviously extended outside the esophagus, the thoracic duct was routinely ligated above the diaphragm.

At the same time, an upper midline abdominal incision was made by another two surgeons, and the abdomen was explored. During mobilization of the stomach, care was taken to preserve the right gastroepiploic vessels and arcades. The left gastric artery and vein were isolated and doubly ligated at their origin. Pyloroplasty was not routinely performed. Then, the hiatus was enlarged and the stomach was pulled into the chest. An endto-side esophagogastric anastomosis was performed within the apex of the chest, and the stomach was secured in the mediastinum

106 (Chen et al. 2009a; Chen et al. 2009b).

\section{Specimens}


The ESCC tissue and ANM (more than $5 \mathrm{~cm}$ from the margin of ESCC) were collected

109 from surgical specimens of each selected patient. At the same time, the ANM did not exhibit

110 tumor infiltration, deterioration or necrosis upon macroscopic and microscopic examination.

This study was approved by the Ethics Committee of Provincial Hospital Affiliated to

112 Shandong University, and the approval number was 2008081. Written informed consent was

113 obtained from all the participants.

\section{Immunohistochemistry}

The streptavidin-peroxidase immunohistochemical method was used to examine the expression of the IFITM3 protein. Formalin-fixed and paraffin-embedded surgical specimens were sequentially cut into $4-\mu \mathrm{m}$ sections. Then, the sections were dewaxed, antigen retrieval and hydrogen peroxide incubation. Rabbit anti-IFITM3 monoclonal antibodies (GeneTex, USA) were used at a dilution of 1:200 and incubated at $4{ }^{\circ} \mathrm{C}$ overnight. The monoclonal primary antibody was replaced by phosphate-buffered saline (PBS) as a negative control. Further experimental steps were followed according to the instructions of a secondary biotinylated antibody kit purchased from ZSGB Biotech (BeiJing, China). 
$127-75 \%)$, and $4(75-100 \%)$. The staining intensity was scored: 0 (negative), 1 (weak), 2

128 (moderate), and 3 (strong). The final immunohistochemical score (IHS) was defined as the

129 proportion score $\times$ staining intensity score. In this study, IHS $\geq 8$ was considered to represent

130 overexpression.

\section{RNA Extraction and RT-PCR}

132

133

134

135

136

137

138

Total RNA was extracted from fresh frozen tissue using Trizol (Invitrogen) according to the manufacturer's protocols. The purity of RNA was measured by UV spectrophotometer (NanoDrop 2000) and the OD 260/280 value ranging from 1.8 to 2.0 was used to reverse transcription. The detailed RT-PCR procedure was followed by a CWBio two-step RT-PCR kit (JiangSu, China). The primer sequences of the IFITM3 gene were 5'-

CAAGGAGGAGCACGAGG-3' (forward primer) and 5'-TTGAACAGGGACCAGACG-3' (reverse primer). $\beta$-actin was used as internal control, and the primer sequences of $\beta$-actin were 5'-AGAGCCTCGCCTTTGCCGATCC-3' (forward primer) and 5'ATACACCCGCTGCTCCGGGTC-3' (reverse primer). The PCR products of IFITM3 were further separated on 1\% agarose gel electrophoresis. Azure C2000 (Azure Biosystems, USA) was used for electrophoresis gelatin image formation analysis.

\section{Follow-Up}

According to our plan, patients were examined every $3-6$ months after surgery, and the checklist was described in our previous study (Akhtar et al. 2014). We compared the imaging 
146 data preoperatively and postoperatively in detail to differentiate whether recurrence occurred or

147 not. If the lymph nodes were swollen or the minor axis was more than $1 \mathrm{~cm}$, the clinical

148 diagnosis of lymphatic recurrence was made. Fine-needle aspiration biospy and PET-CT were

149 also assisted to make the diagnosis of recurrence. The follow-up was ended in July 2013, and the

150 complete 3-year follow-up data was reviewed.

\section{Statistical Analysis}

The $\chi^{2}$ test was used to analyze the relationship between IFITM3 expression and

153

154

155

156

157

158

clinicopathological variables. The recurrence curves and survival curves were calculated by the Kaplan-Meier method. Univariate log-rank test and Cox regression analysis were respectively performed to evaluate the recurrence risk and prognostic factors. A satatistically significant difference was represented by a two-tailed $p$ value less than 0.05 . All statistical analyses were performed using SPSS version 17.0 (Chicago, IL, USA).

\section{Results}

\section{IFITM3 Expression Analysis in ESCC tissue and ANM}

The immunohistochemistry assay was used to detect the expression level of the IFITM3

protein. Overexpression was presented as yellow or brownish yellow staining in the cytoplasm of the tumor cell. As is shown in Fig. 1C, the significant immunoreaction of positive expression can be readily differentiated. However, there was low or undetected staining in ANM (Fig. 1A).

Furthermore, according to the criteria of IHS, we divided all the specimens into two groups: 59 
165

166

167

168

169

170

171

cases $(56.7 \%)$ were categorized as the overexpression group (Fig. 1C) and 45 cases (43.3\%) were in the low expression group (Fig. 1B).

To verify this aberrant upregulation of IFITM3, we examined the mRNA expression level by RT-PCR with 20 pairs of specimens randomly selected from the overexpression group and 20 pairs of tissues that originated from the low expression group. The results showed that the mRNA expression level was consistent with protein expression as demonstrated by IHC (Fig. 2).

\section{IFITM3 Expression and Clinicopathological Characteristics}

enrolled in this study with different ages, genders, tumor sizes, degrees of differentiation, $\mathrm{T}$

status and IFITM3 expression levels (Table 1). $\chi^{2}$ analysis demonstrated that the expression level of IFITM3 had a close relationship with T status of tumor $(p=0.015)$. In contrast, there were no significant differences between expression level and age, gender, tumor size and degree of differentiation. $(p>0.05)$

\section{IFITM3 Expression and Lymphatic Metastatic Recurrence}

Through thorough follow-up, a total of 42 cases (40.4\%) were confirmed to have first lymph node metastatic recurrence within 3 years, in which IFITM3 overexpression was detected in 30 patients (71.4\%). In the low IFITM3 expression group, the 3-year lymphatic recurrence rate was only $26.7 \%$. Conversely, in the overexpression group, this rate reached up to $50.8 \%$ (Table 1). As is shown in Fig. 3, Kaplan-Meier analysis showed that the recurrence rate was 
184 significantly increased in patients with IFITM3 overexpression, and the log-rank test calculated

185 that these two curves were significantly different $(p=0.010)$.

186

187

188

189

190

In addition to the expression level of IFITM3, T status of tumor $(p=0.008)$ was also elucidated to be associated with the lymphatic recurrence in pN0 ESCC (Table 1). Multivariate Cox regression analysis revealed that these two variables were independent recurrence risk factors $(p<0.05$, Table 2). Patients with early T status and low expression of IFITM3 may have a lower recurrence risk of lymphatic metastasis (Fig. 3).

\section{IFITM3 Expression and Overall Survival}

As is shown in our data, the 3-year OS rate of patients with IFITM3 protein overexpression and low expression were respectively $64.4 \%$ and $88.9 \%$. Fig. 4 demonstrated that the long survival of patients was associated with moderate-high differentiation, early $\mathrm{T}$ status of tumor and low IFITM3 expression. Univariate analysis and multivariate analysis revealed that these are independent and significant prognostic factors $(p<0.05$, Table 3).

\section{Discussion}

ESCC is one of the most common neoplasms in China, with a high incidence of lymph node metastatic recurrence, especially in the mediastinum, neck and abdominal cavity (Chen et al. 2007). Even in pN0 ESCC, more than $40 \%$ of individuals exhibit micro-metastasis (Wang et al. 2004). Surgery is still considered to be the first-line treatment modality for ESCC patients with resectable lesions (Hulscher et al. 2002; Olsen et al. 2011), but the overall survival is not ideal 
203

204

and nearly half of patients will still experience tumor relapse (Eloubeidi et al. 2002; Korst et al. 1998; Rice et al. 2001; Visbal et al. 2001). cancer guidelines are often referenced in clinical practice. These guidelines suggest that patients should not receive adjuvant therapy after complete tumor resection, but individuals with advanced T status (above T2) should receive neoadjuvant chemotherapy before surgery.

However, in China, patients tend to receive primary surgery if tumors can be completely resected and Ivor-Lewis esophagectomy via a thoracoabdominal two-field lymph node dissection is the main surgical modality. Compared with three-field lymph node dissection, the advantage of IvorLewis esophagectomy is that the latent surgical trauma and complications can be effectively controlled; however, cervical lymph node dissection cannot be accomplished simultaneously. Therefore, for patients with a high risk of lymphatic metastasis, we believe that postoperative adjuvant therapy may act as a compensatory mechanism to control lymphatic recurrence after Ivor-Lewis esophagectomy.

In this study, we first found the differential expression of IFITM3 in tumor tissues and their ANMs, as well as the important clinicopathological significance of IFITM3. Our results were consistent with previous researches that demonstrated that IFITM3 is overexpressed in many hunman malignancies, such as gastric cancer, colorectal cancer, oral squamous cell carcinoma, glioma, and breast cancer. These findings suggested that IFITM3 may play important roles and maybe a molecular marker in pN0 ESCC. 
conclusions for different cancers, which reflects the complexity of IFITM3 in different tumor

225

226

227

228

229

230

231

232

233

234

235

236

microenvironments. For gastric cancer, $\mathrm{Hu}$ et al (Hu et al. 2014) suggested that IFITM3

overexpression is correlated with lymph node metastasis. Li et al demonstrated that it was an important independent prognostic factor for disease-free interval and is upregulated in the nodal metastasis of colon tumors (Li et al. 2011). Conversely, Yang et al did not find an association between IFITM3 expression and lymph node metastasis in breast cancer (Yang et al. 2013). ElTanani et al (El-Tanani et al. 2010) even drew the opposite conclusion; they deemed that IFITM3 may inhibit the proliferation, development and metastasis of cancer by reducing the expression of osteopontin. However, to our knowledge, no study has demonstrated the prognostic significance of IFITM3 in ESCC. This question led us to explore whether IFITM3 could be a biomarker to evaluate the risk of lymph node metastatic recurrence in ESCC. The data from this study showed that the high incidence of lymphatic metastatic recurrence in pNO ESCC was associated with advanced T status and IFITM3 overexpression. At the same time, the expression level of IFITM3 was also elucidated to be an important prognostic factor. The findings strongly suggested that IFITM3 could serve as a biomarker to stratify the risk of recurrence and survival, and then play an important role in the selection of a treatment modality in pN0 ESCC.

$$
\text { esophagectomy with two-field lymph node dissection. All of these patients had undergone }
$$

theoretic R0 resection and had pathologically confirmed pN0 after surgery. However, during the 
243 follow-up period, 40.4\% patients showed the first lymphatic metastatic recurrence within 3 years.

244 For patients with a high risk of lymphatic metastatic recurrence, we believe that these findings

245 have important clinical significance in choosing to accept adjuvant therapy to control lymph

246 node recurrence. Previous studies have demonstrated that postoperative adjuvant radiotherapy

247 can significantly reduce the lymphatic metastatic recurrence in ESCC. Combined with findings

248 in this study and our previous studies, we believe that it is indispensible for pN0 ESCC patients

249 with IFITM3 overexpression to receive postoperative adjuvant radiotherapy to control the

250 metastatic recurrence of locoregional lymph nodes and in turn improve the survival.

However, this study was retrospective and had a limited sample size. Although this is the

252 first time that IFITM3 was demonstrated to be a predictor for lymph node metastatic recurrence

253 of ESCC patients, replication studies with different parameters and prospective and multicenter

254 randomized studies are also needed to verify this prognostic significance.

\section{Conclusions}

Our study demonstrated that IFITM3 expression has a close relationship with lymphatic metastatic recurrence. This could serve as an important biomarker to predict the lymph node metastatic recurrence in pNO ESCC after Ivor-Lewis esophagectomy.

Conflict of interest The authors declare that they have no conflicts of interest. 
263

264

265

266

Akhtar J, Wang Z, Yu C, Zhang ZP, and Bi MM. 2014. STMN-1 gene: a predictor of survival in stage iia esophageal squamous cell carcinoma after Ivor-Lewis esophagectomy? Ann Surg Oncol 21:315-321.

Arai J, Tsuchiya T, Oikawa M, Mochinaga K, Hayashi T, Yoshiura K, Tsukamoto K, Yamasaki N, Matsumoto K, Miyazaki T, and Nagayasu T. 2012. Clinical and molecular analysis of synchronous double lung cancers. Lung Cancer 77:281-287.

Baba M, Aikou T, Natsugoe S, Kusano C, Shimada M, Nakano S, Fukumoto T, and Yoshinaka H. 1998. Quality of life following esophagectomy with three-field lymphadenectomy for carcinoma, focusing on its relationship to vocal cord palsy. Dis Esophagus 11:28-34.

Chen G, Wang Z, Liu XY, and Liu FY. 2007. Recurrence pattern of squamous cell carcinoma in the middle thoracic esophagus after modified Ivor-Lewis esophagectomy. World J Surg 31:1107-1114.

Chen G, Wang Z, Liu XY, and Liu FY. 2009a. Adjuvant radiotherapy after modified Ivor-Lewis esophagectomy: can it prevent lymph node recurrence of the mid-thoracic esophageal carcinoma? Ann Thorac Surg 87:1697-1702.

Chen G, Wang Z, Liu XY, Zhang MY, and Liu FY. 2009b. Clinical study of modified IvorLewis esophagectomy plus adjuvant radiotherapy for local control of stage IIA squamous cell carcinoma in the mid-thoracic esophagus. Eur J Cardiothorac Surg 35:1-7.

El-Tanani MK, Jin D, Campbell FC, and Johnston PG. 2010. Interferon-induced transmembrane 3 binds osteopontin in vitro: expressed in vivo IFITM3 reduced OPN expression. Oncogene 29:752-762.

Eloubeidi MA, Desmond R, Arguedas MR, Reed CE, and Wilcox CM. 2002. Prognostic factors for the survival of patients with esophageal carcinoma in the U.S.: the importance of tumor length and lymph node status. Cancer 95:1434-1443.

Ferlay J, Shin HR, Bray F, Forman D, Mathers C, and Parkin DM. 2010. Estimates of worldwide burden of cancer in 2008: GLOBOCAN 2008. Int J Cancer 127:2893-2917.

Gu Y, Swisher SG, Ajani JA, Correa AM, Hofstetter WL, Liao Z, Komaki RR, Rashid A, Hamilton SR, and Wu TT. 2006. The number of lymph nodes with metastasis predicts 
survival in patients with esophageal or esophagogastric junction adenocarcinoma who receive preoperative chemoradiation. Cancer 106:1017-1025.

Hu J, Wang S, Zhao Y, Guo Q, Zhang D, Chen J, Li J, Fei Q, and Sun Y. 2014. Mechanism and biological significance of the overexpression of IFITM3 in gastric cancer. Oncol Rep 32:2648-2656.

Hulscher JB, van Sandick JW, de Boer AG, Wijnhoven BP, Tijssen JG, Fockens P, Stalmeier PF, ten Kate FJ, van Dekken H, Obertop H, Tilanus HW, and van Lanschot JJ. 2002. Extended transthoracic resection compared with limited transhiatal resection for adenocarcinoma of the esophagus. N Engl J Med 347:1662-1669.

Korst RJ, Rusch VW, Venkatraman E, Bains MS, Burt ME, Downey RJ, and Ginsberg RJ. 1998. Proposed revision of the staging classification for esophageal cancer. J Thorac Cardiovasc Surg 115:660-669; discussion 669-670.

Law S, and Wong J. 2001. Two-field dissection is enough for esophageal cancer. Dis Esophagus 14:98-103.

Li D, Peng Z, Tang H, Wei P, Kong X, Yan D, Huang F, Li Q, Le X, Li Q, and Xie K. 2011. KLF4-mediated negative regulation of IFITM3 expression plays a critical role in colon cancer pathogenesis. Clin Cancer Res 17:3558-3568.

Li SH, Wang Z, and Liu XY. 2009. Metastasis-associated protein 1 (MTA1) overexpression is closely associated with shorter disease-free interval after complete resection of histologically node-negative esophageal cancer. World J Surg 33:1876-1881.

Matsubara T, Ueda M, Takahashi T, Nakajima T, and Nishi M. 1996. Localization of recurrent disease after extended lymph node dissection for carcinoma of the thoracic esophagus. $J$ Am Coll Surg 182:340-346.

Nakagawa S, Kanda T, Kosugi S, Ohashi M, Suzuki T, and Hatakeyama K. 2004. Recurrence pattern of squamous cell carcinoma of the thoracic esophagus after extended radical esophagectomy with three-field lymphadenectomy. J Am Coll Surg 198:205-211.

Natsugoe S, Yoshinaka H, Shimada M, Sakamoto F, Morinaga T, Nakano S, Kusano C, Baba M, Takao S, and Aikou T. 2001. Number of lymph node metastases determined by presurgical ultrasound and endoscopic ultrasound is related to prognosis in patients with esophageal carcinoma. Ann Surg 234:613-618. 
321

Olsen JR, Dehdashti F, Siegel BA, Zighelboim I, Grigsby PW, and Schwarz JK. 2011. Prognostic utility of squamous cell carcinoma antigen in carcinoma of the cervix: association with pre- and posttreatment FDG-PET. Int J Radiat Oncol Biol Phys 81:772777.

Rice TW, Blackstone EH, Adelstein DJ, Zuccaro G, Jr., Vargo JJ, Goldblum JR, Rybicki LA, Murthy SC, and Decamp MM. 2001. N1 esophageal carcinoma: the importance of staging and downstaging. J Thorac Cardiovasc Surg 121:454-464.

Song Y, Wang Z, Liu X, Jiang W, and Shi M. 2012. CCR7 and VEGF-C: molecular indicator of lymphatic metastatic recurrence in pN0 esophageal squamous cell carcinoma after IvorLewis esophagectomy? Ann Surg Oncol 19:3606-3612.

Visbal AL, Allen MS, Miller DL, Deschamps C, Trastek VF, and Pairolero PC. 2001. Ivor Lewis esophagogastrectomy for esophageal cancer. Ann Thorac Surg 71:1803-1808.

Wang Z, Liu XY, Liu FY, and Chen JH. 2004. [A study of correlation between early postoperative relapse with lymph node micrometastasis in patients with N0 esophageal cancer]. Zhonghua Wai Ke Za Zhi 42:68-71.

Yang M, Gao H, Chen P, Jia J, and Wu S. 2013. Knockdown of interferon-induced transmembrane protein 3 expression suppresses breast cancer cell growth and colony formation and affects the cell cycle. Oncol Rep 30:171-178.

Zhao B, Wang H, Zong G, and Li P. 2013. The role of IFITM3 in the growth and migration of human glioma cells. BMC Neurol 13:210. 
Table $\mathbf{1}$ (on next page)

Correlation of IFITM3 expression with clinicopathological characteristics

Table1: Correlation of IFITM3 expression with clinicopathological characteristics of pNO ESCC patients 


\begin{tabular}{|c|c|c|c|c|c|c|}
\hline \multirow{3}{*}{ Variables } & \multirow{3}{*}{$\begin{array}{l}\text { No. of } \\
\text { patients }\end{array}$} & \multicolumn{2}{|c|}{ IFITM3 Expression } & \multirow{3}{*}{$p$ value $^{\mathrm{a}}$} & \multirow{3}{*}{3 -year recurrence rate $(\%)$} & \multirow{3}{*}{$p$ value ${ }^{b}$} \\
\hline & & High & Low & & & \\
\hline & & 59 & 45 & & & \\
\hline Age (years) & & & & 0.335 & & 0.550 \\
\hline$\geq 50$ & 83 & 45 & 38 & & 42.2 & \\
\hline$<50$ & 21 & 14 & 7 & & 33.3 & \\
\hline Gender & & & & 0.365 & & 0.889 \\
\hline Male & 78 & 42 & 36 & & 41.0 & \\
\hline Female & 26 & 17 & 9 & & 38.5 & \\
\hline Tumor size $(\mathrm{cm})$ & & & & 0.418 & & 0.193 \\
\hline$\geq 5$ & 40 & 25 & 15 & & 47.5 & \\
\hline
\end{tabular}




\begin{tabular}{|c|c|c|c|c|c|c|}
\hline$<5$ & 64 & 34 & 30 & & 35.9 & \\
\hline $\mathrm{T}$ status & & & & 0.015 & & 0.008 \\
\hline $\mathrm{T} 1+\mathrm{T} 2$ & 41 & 30 & 11 & & 24.4 & \\
\hline $\mathrm{T} 3$ & 55 & 24 & 31 & & 49.1 & \\
\hline $\mathrm{T} 4 \mathrm{a}$ & 8 & 5 & 3 & & 62.5 & \\
\hline Differentiation degree & & & & 0.249 & & 0.111 \\
\hline Low & 25 & 17 & 8 & & 52.0 & \\
\hline Moderate-High & 79 & 42 & 37 & & 36.7 & \\
\hline IFITM3 overexpression & & & & & & 0.010 \\
\hline Yes & & 59 & & & 50.8 & \\
\hline No & & & 45 & & 26.7 & \\
\hline
\end{tabular}




\section{PeerJ}

1 a $\chi^{2}$ test

2 b Log-rank test

3 
Table 2 (on next page)

Multivariate Cox regression analysis

Table 2: Multivariate Cox regression analysis of risk factors in pNO ESCC 


\begin{tabular}{|c|c|c|c|c|c|c|c|}
\hline & \multirow{2}{*}{ B } & \multirow{2}{*}{$\mathrm{SE}$} & \multirow{2}{*}{ Wald } & \multirow{2}{*}{$p$ value } & \multirow{2}{*}{ HR } & \multicolumn{2}{|c|}{$95 \% \mathrm{CI}$} \\
\hline & & & & & & Lower & Upper \\
\hline Age & 0.040 & 0.453 & 0.008 & 0.930 & 1.041 & 0.429 & 2.526 \\
\hline Gender & 0.041 & 0.389 & 0.011 & 0.915 & 1.042 & 0.487 & 2.232 \\
\hline Tumor size & 0.226 & 0.324 & 0.489 & 0.484 & 1.254 & 0.665 & 2.365 \\
\hline T status & 0.904 & 0.253 & 12.783 & 0.000 & 2.470 & 1.505 & 4.054 \\
\hline Differentiation & 0.311 & 0.350 & 0.790 & 0.374 & 1.365 & 0.687 & 2.712 \\
\hline IFITM3 overexpression & 1.040 & 0.360 & 8.357 & 0.004 & 2.828 & 1.398 & 5.723 \\
\hline
\end{tabular}

1 B regression coefficient; SE standard error; Wald Wald value; HR hazard ratio; CI confidence interval 


\section{Table 3(on next page)}

Univariate and multivariate analyses of overall survival

Table 3: Univariate and multivariate analyses of overall survival for 104 cases of pNO ESCC patients 


\begin{tabular}{|c|c|c|c|c|c|c|}
\hline \multirow[b]{2}{*}{ Variables } & \multicolumn{3}{|c|}{ Univariate analysis } & \multicolumn{3}{|c|}{ Multivariate analysis } \\
\hline & HR & $95 \% \mathrm{CI}$ & $p$ & HR & $95 \% \mathrm{CI}$ & $p$ \\
\hline \multicolumn{7}{|l|}{ Gender } \\
\hline Male versus Female & 1.095 & $0.440-2.727$ & 0.846 & - & & \\
\hline \multicolumn{7}{|l|}{ Age (years) } \\
\hline$\geq 50$ versus $<50$ & 1.388 & $0.478-4.028$ & 0.546 & - & & \\
\hline \multicolumn{7}{|l|}{ Tumor size $(\mathrm{cm})$} \\
\hline$\geq 5$ versus $<5$ & 2.076 & $0.960-4.490$ & 0.063 & _- & & \\
\hline \multicolumn{7}{|l|}{$\mathrm{T}$ status } \\
\hline $\mathrm{T} 1+\mathrm{T} 2$ versus $\mathrm{T} 3+\mathrm{T} 4 \mathrm{a}$ & 3.082 & $1.162-8.175$ & 0.024 & 3.979 & $1.443-10.974$ & 0.008 \\
\hline \multicolumn{7}{|l|}{ Differentiation degree } \\
\hline Moderate-High versus Low & 3.125 & $1.445-6.758$ & 0.004 & 2.346 & $1.056-5.212$ & 0.036 \\
\hline \multicolumn{7}{|l|}{ IFITM3 overexpression } \\
\hline Yes versus No & 3.644 & $1.373-9.673$ & 0.009 & 4.260 & $1.548-11.723$ & 0.005 \\
\hline
\end{tabular}


2 Statistical analysis was performed using the proportional hazard model (Cox). Data considered significant $(p<0.05)$ in the univariate 3 analysis were examined in the multivariate analysis

4 HR hazard ratio; CI confidence interval

$5 \quad p$ values $<0.05$ in bold font were considered significant 


\section{1}

Immunohistochemistry assay of IFITM3 in ESCC tissue and ANM

Figure 1: Immunohistochemistry assay of IFITM3 in ESCC tissue and ANM. (A) Negative expression of IFITM3 in ANM $(\times 200, \times 400)$. (B) Low expression in ESCC tissue $(\times 200, \times 400)$. (C) Strong positive immunocreation of IFITM3 in the cytoplasm of ESCC tissue $(\times 200, \times 400)$

A
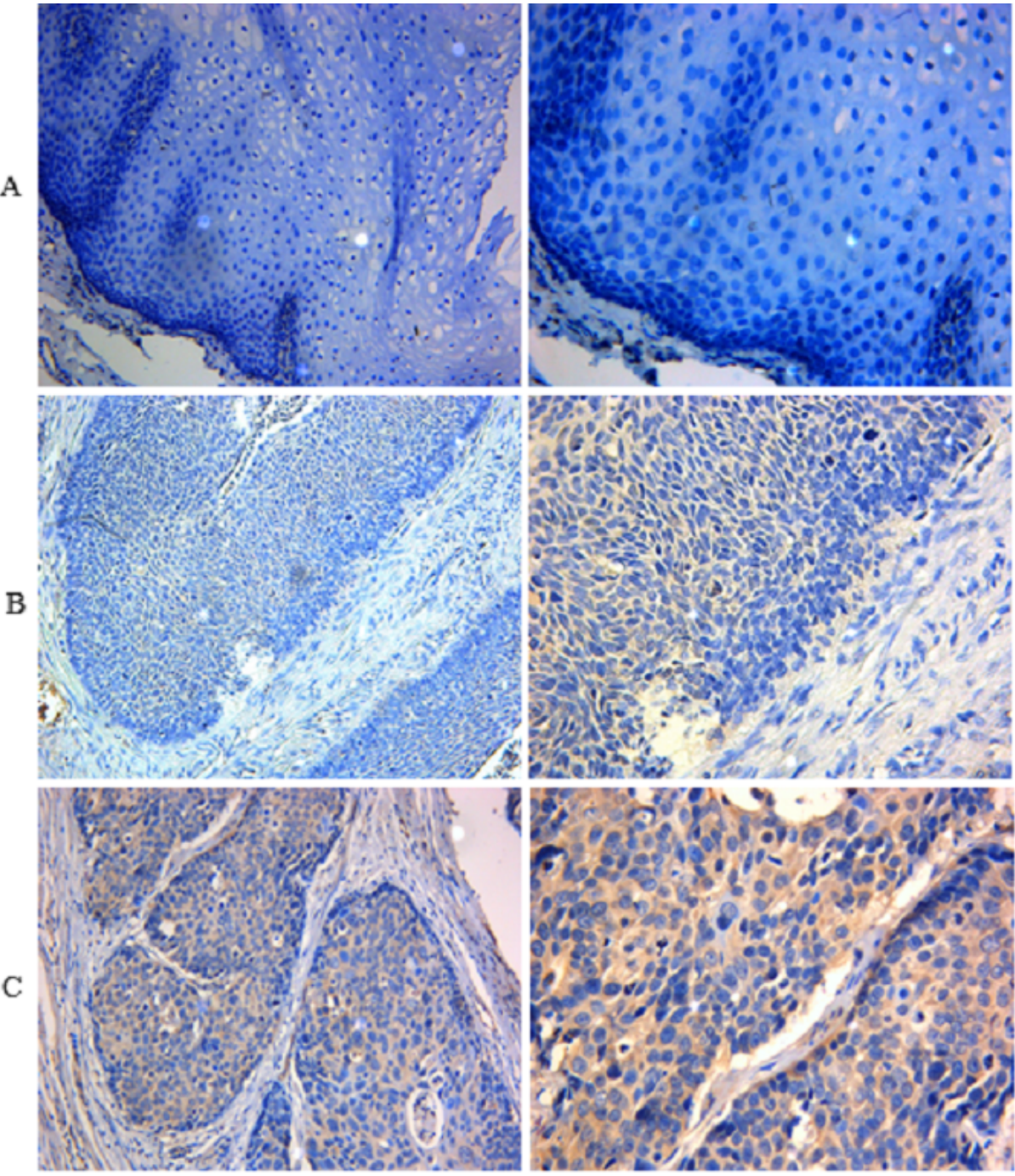

$\times 200$

$\times 400$ 
2

Relative expression level of IFITM3 mRNA was detected by RT-PCR

Figure 2: (M) Molecular marker. (1) and (2) Respectively represent the mRNA expression level of $\beta$-actin and IFITM3 in tumor tissues with IFITM3 protein overexpression; (3) and (4) Represent the mRNA expression level of $\beta$-actin and IFITM3 in their ANMs. (5) and (6)

Respectively represent the mRNA expression level of $\beta$-actin and IFITM3 in low IFITM3 protein expressed tumor tissues; (7) and (8) Represent this expression level of $\beta$-actin and IFITM3 in their ANMs

$\begin{array}{lllllllll}M & 1 & 2 & 3 & 4 & 5 & 6 & 7 & 8\end{array}$

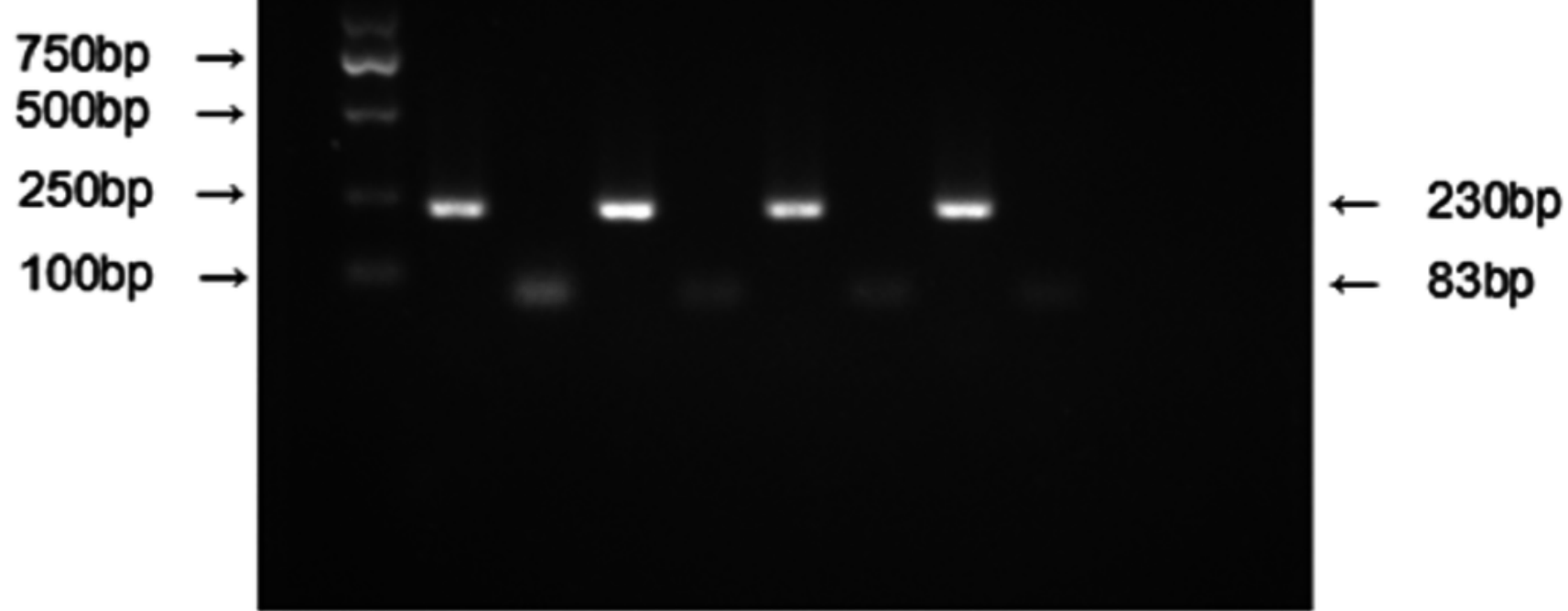


3

Lymphatic metastatic recurrence curves for patients with different IFITM3 expression level and T status

Figure 3: Lymphatic metastatic recurrence curves for patients with different IFITM3 expression level and T status. (A) and (B) Respectively represent patients with IFITM3 overexpression $(p=0.010)$ and advanced T status $(p=0.004)$

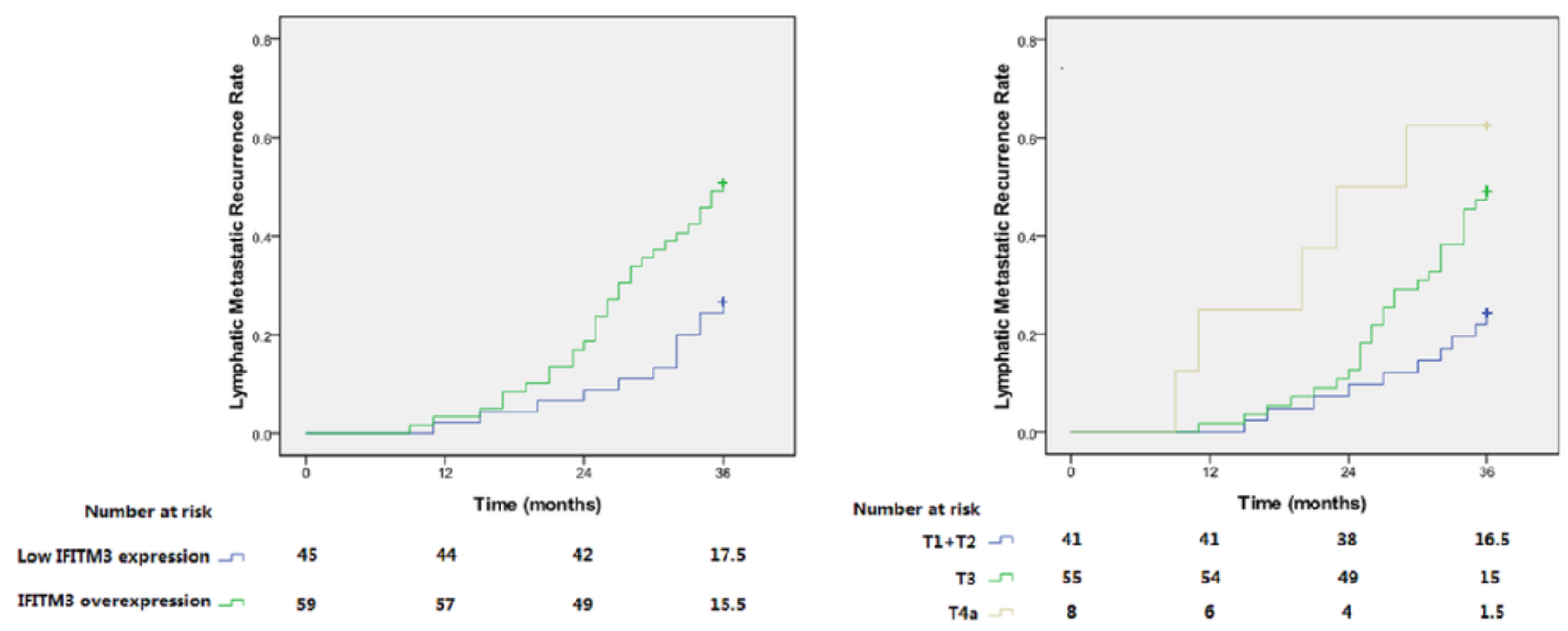


4

Kaplan-Meier analysis of overall survival of ESCC patients

Figure 4: Kaplan-Meier analysis of overall survival of ESCC patients. Overall survival of patients with pNO ESCC according to (A) IFITM3 expression level; (B) T status of tumor; (C) differentiation degree
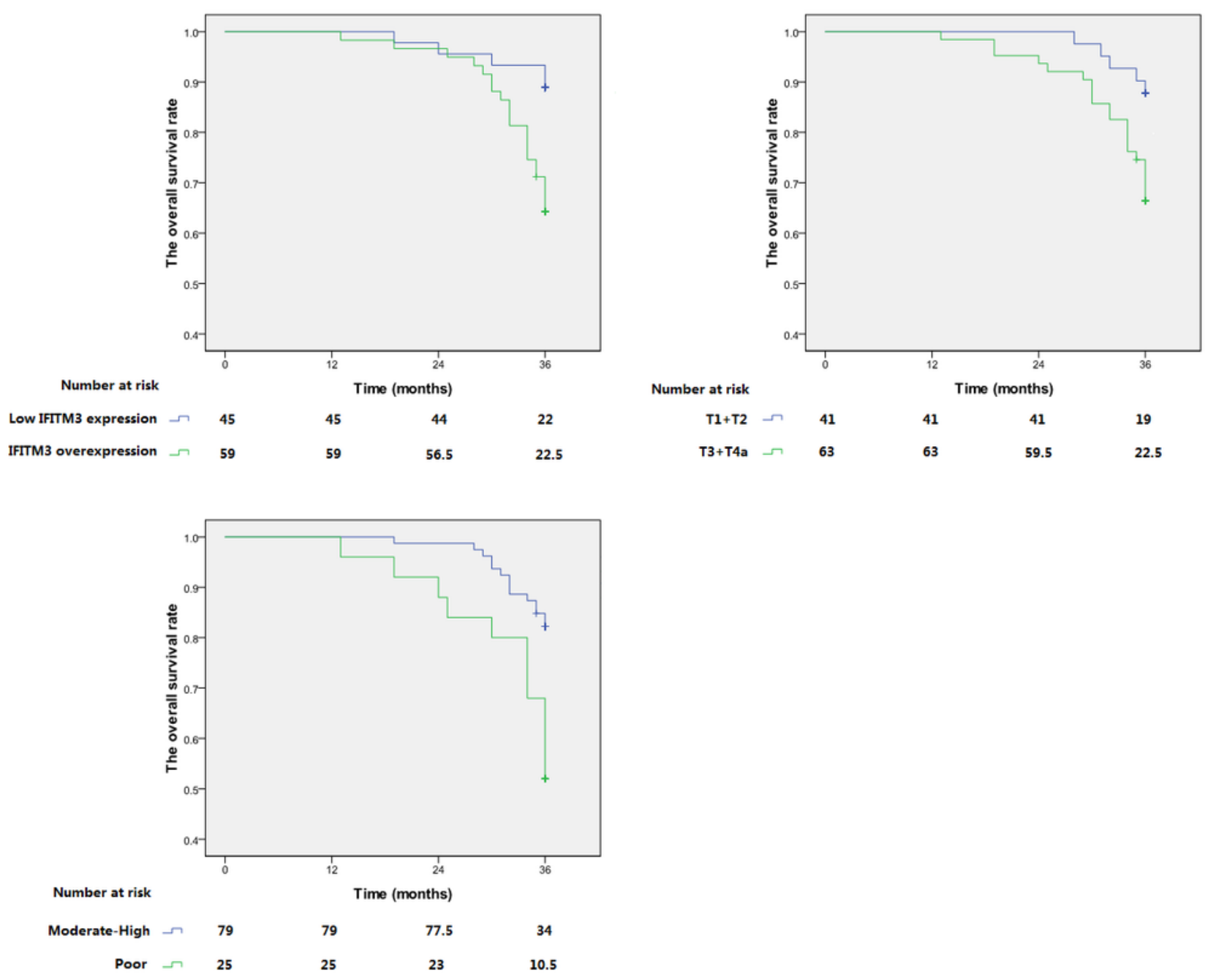